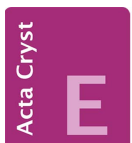
COMMUNICATIONS

ISSN 2056-9890

\section{Crystal structure of 3-acetyl-4H- chromen-4-one}

\section{Yoshinobu Ishikawa}

School of Pharmaceutical Sciences, University of Shizuoka, 52-1 Yada, Suruga-ku, Shizuoka 422-8526, Japan. *Correspondence e-mail: ishi206@u-shizuoka-ken.ac.jp

Received 22 June 2015; accepted 24 June 2015

Edited by W. T. A. Harrison, University of Aberdeen, Scotland

In the title compound, $\mathrm{C}_{11} \mathrm{H}_{8} \mathrm{O}_{3}$, the fused-ring system is almost planar (r.m.s. deviation $=0.020 \AA$ ), with the largest deviation from the least-squares plane [0.0462 (17) $\AA$ ] being for a pyran $\mathrm{C}$ atom. The dihedral angle between the plane of the fused-ring system and acetyl plane is $5.149(16)^{\circ}$. In the crystal, the fused rings are linked by aromatic $\pi-\pi$ stacking interactions [centroid-centroid distance between the benzene and pyran rings $=3.643(6) \AA]$ and $\mathrm{C}-\mathrm{H} \cdots \mathrm{O}$ hydrogen bonds, generating a three-dimensional network.

Keywords: crystal structure; chromone; hydrogen bond; $\pi-\pi$ stacking.

CCDC reference: 1408496

\section{Related literature}

For a related structure, see: Chanda et al. (2014). For further synthetic details, see: Yokoe et al. (1994); Li et al. (2012).<smiles>CC(=O)c1coc2ccccc2c1=O</smiles>

\section{Experimental}

\subsection{Crystal data}

$\mathrm{C}_{11} \mathrm{H}_{8} \mathrm{O}_{3}$

$M_{r}=188.18$

Monoclinic, $P 2_{1} / n$

$$
\begin{aligned}
& a=8.016(13) \AA \\
& b=25.93(6) \AA \\
& c=4.091(8) \AA
\end{aligned}
$$

$$
\begin{aligned}
& \beta=94.79(14)^{\circ} \\
& V=847(3) \AA^{3} \\
& Z=4
\end{aligned}
$$$$
\text { Mo } K \alpha \text { radiation }
$$

$\mu=0.11 \mathrm{~mm}^{-1}$

$T=100 \mathrm{~K}$

$0.42 \times 0.25 \times 0.20 \mathrm{~mm}$

\subsection{Data collection}

Rigaku AFC-7R diffractometer 2377 measured reflections 1962 independent reflections 1510 reflections with $F^{2}>2.0 \sigma\left(F^{2}\right)$

$R_{\text {int }}=0.018$

3 standard reflections every 150 reflections intensity decay: $-0.5 \%$

\subsection{Refinement}

$R\left[F^{2}>2 \sigma\left(F^{2}\right)\right]=0.041$

$w R\left(F^{2}\right)=0.112$

$S=1.03$

1959 reflections

128 parameters

$\mathrm{H}$-atom parameters constrained

$\Delta \rho_{\max }=0.31 \mathrm{e}^{-3}$

$\Delta \rho_{\min }=-0.20 \mathrm{e}^{-3}$

Table 1

Hydrogen-bond geometry $\left(\AA,^{\circ}\right)$.

\begin{tabular}{lllll}
\hline$D-\mathrm{H} \cdots A$ & $D-\mathrm{H}$ & $\mathrm{H} \cdots A$ & $D \cdots A$ & $D-\mathrm{H} \cdots A$ \\
\hline $\mathrm{C} 7-\mathrm{H} 5 \cdots \mathrm{O} 2^{\mathrm{i}}$ & 0.95 & 2.40 & $3.292(6)$ & 155 \\
$\mathrm{C} 1-\mathrm{H} 1 \cdots \mathrm{O} 3^{\text {ii }}$ & 0.95 & 2.31 & $3.264(5)$ & 148 \\
\hline
\end{tabular}

Symmetry codes: (i) $x-1, y, z$; (ii) $-x,-y,-z+3$.

Data collection: WinAFC Diffractometer Control Software (Rigaku, 1999); cell refinement: WinAFC Diffractometer Control Software; data reduction: WinAFC Diffractometer Control Software; program(s) used to solve structure: SIR2008 (Burla, et al., 2007); program(s) used to refine structure: SHELXL97 (Sheldrick, 2008); molecular graphics: CrystalStructure (Rigaku, 2010); software used to prepare material for publication: CrystalStructure.

\section{Acknowledgements}

The University of Shizuoka is acknowledged for instrumental support.

Supporting information for this paper is available from the IUCr electronic archives (Reference: HB7454).

\section{References}

Burla, M. C., Caliandro, R., Camalli, M., Carrozzini, B., Cascarano, G. L., De Caro, L., Giacovazzo, C., Polidori, G., Siliqi, D. \& Spagna, R. (2007). J. Appl. Cryst. 40, 609-613.

Chanda, T., Chowdhury, S., Koley, S., Anand, N. \& Singh, M. S. (2014). Org. Biomol. Chem. 12, 9216-9222.

Li, G., Zhang, Z. T., Dai, L. Y., Du, Y. L. \& Xue, D. (2012). Helv. Chim. Acta, 95, 989-997.

Rigaku (1999). WinAFC Diffractometer Control Software. Rigaku Corporation, Tokyo, Japan.

Rigaku (2010). CrystalStructure. Rigaku Corporation, Tokyo, Japan.

Sheldrick, G. M. (2008). Acta Cryst. A64, 112-122.

Yokoe, I., Maruyama, K., Sugita, Y., Harashida, T. \& Shirataki, Y. (1994). Chem. Pharm. Bull. 42, 1697-1699. 


\section{supporting information}

Acta Cryst. (2015). E71, o527 [doi:10.1107/S2056989015012098]

\section{Crystal structure of 3-acetyl-4H-chromen-4-one}

\section{Yoshinobu Ishikawa}

\section{S1. Comment}

Many derivatives of the title compound are reported because of their chemical, biological and medicinal significance (Yokoe et al. 1994, Chanda et al. 2014).

The mean deviation of the least-square plane for the non-hydrogen atoms of the fused-ring is $0.0201 \AA$, and the largest deviation from the plane is 0.0462 (17) $\AA$ for $\mathrm{C} 2$. These mean that these atoms are essentially coplanar (Fig.1). The dihedral angle between the fused-ring and acetyl plane is 5.149 (16) $\AA$.

In the crystal, the molecules are linked by $\pi-\pi$ stacking [centroid-centroid distance between the benzene and pyran rings $=3.643$ (6) $\AA$ ] , and $\mathrm{C}-\mathrm{H} \cdots \mathrm{O}$ hydrogen bonds form sheets along [0 4 1] and [0 4 1] ], as shown in Fig.2 and Fig.3.

The crystal structure of a 2,5,6,7-substituted 3-acetylchromone derivative is reported (Chanda et al. 2014).

\section{S2. Experimental}

The title compound was synthesized from 3-(dimethylamino)-1-(2-hydroxyphenyl)prop-2-enone (Li et al. 2012) according to the literature method (Yokoe et al. 1994). Single crystals suitable for X-ray diffraction were obtained by slow evaporation of an ethyl acetate solution of the title compound at room temperature.

\section{S3. Refinement}

All hydrogen atoms were placed in geometrical positions [C-H 0.95 and $0.98 \AA]$, and refined using a riding model with $U_{\text {iso }}(\mathrm{H})=1.2 U_{\text {eq }}$ of the parent atoms. The s.u.s for the cell parameters are rather large, possibly due to frost damage to the crystal. 


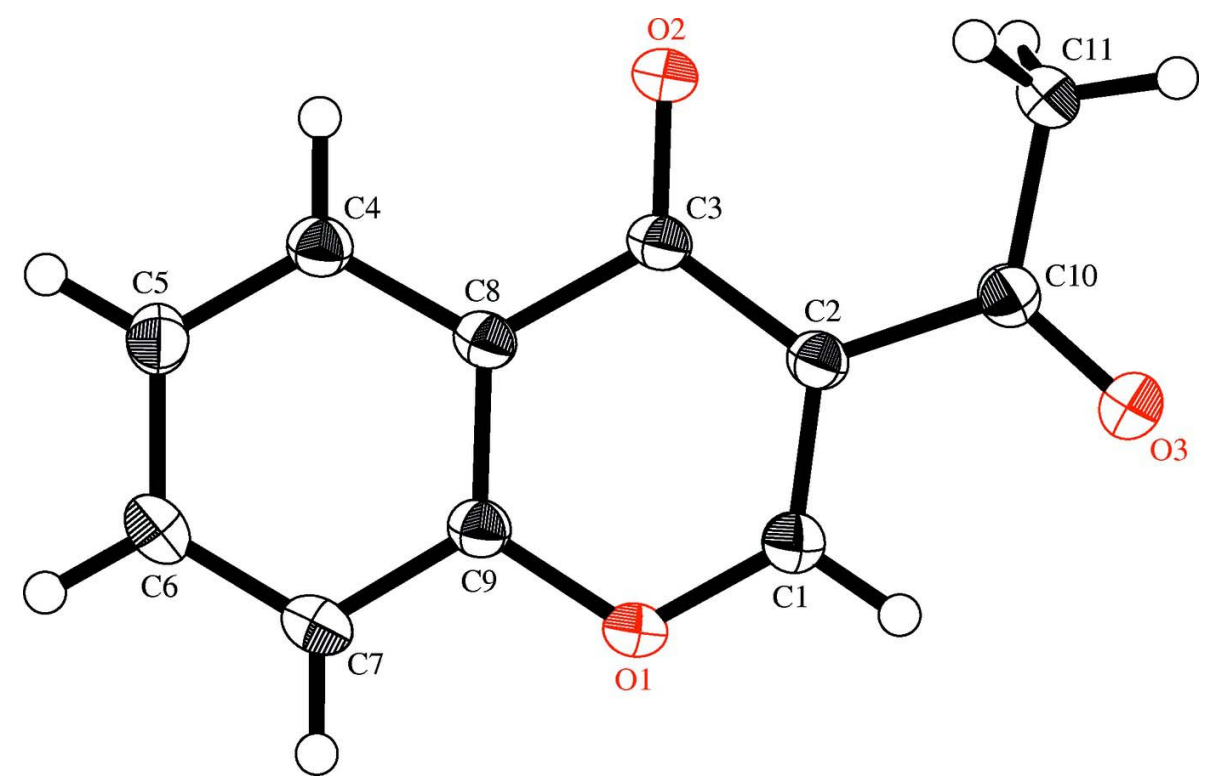

\section{Figure 1}

The molecular structure of the title compound, with displacement ellipsoids drawn at the $50 \%$ probability level. Hydrogen atoms are shown as small spheres of arbitrary radius.

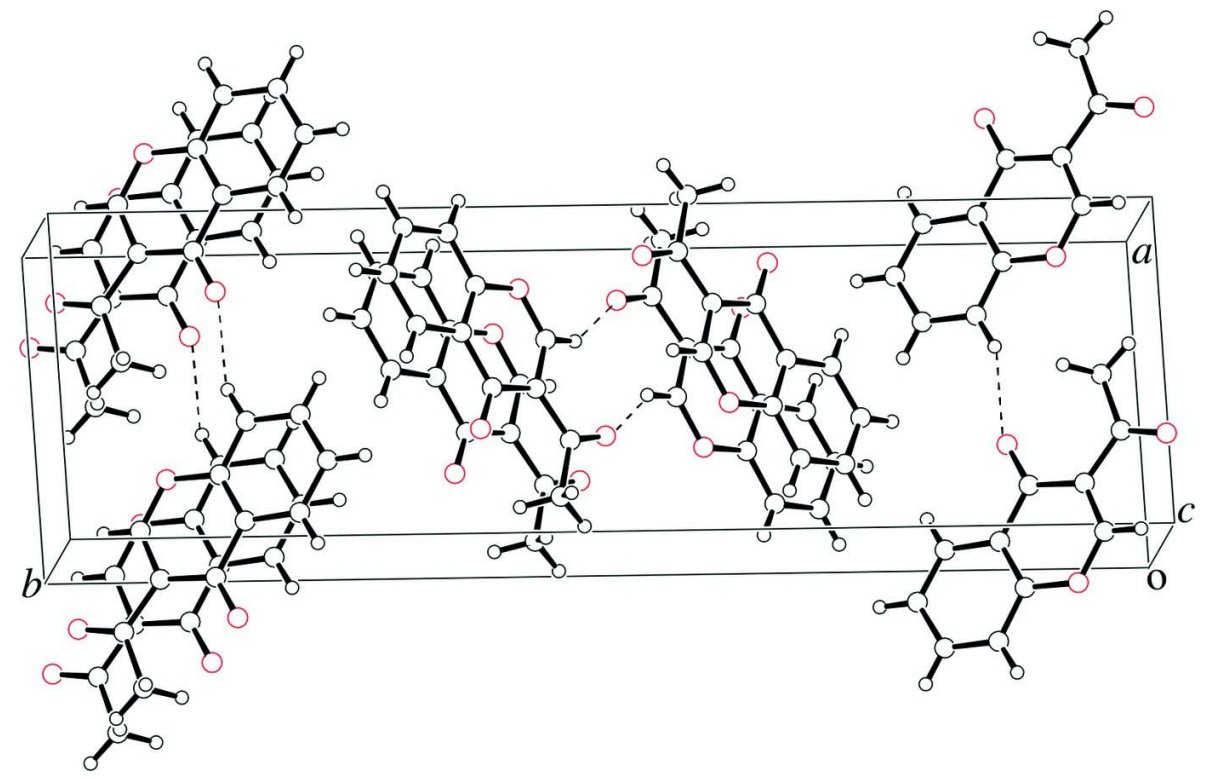

Figure 2

A view of the intermolecular interactions of the title compound. $\mathrm{C}-\mathrm{H} \cdots \mathrm{O}$ hydrogen bonds are represented as dashed lines. 


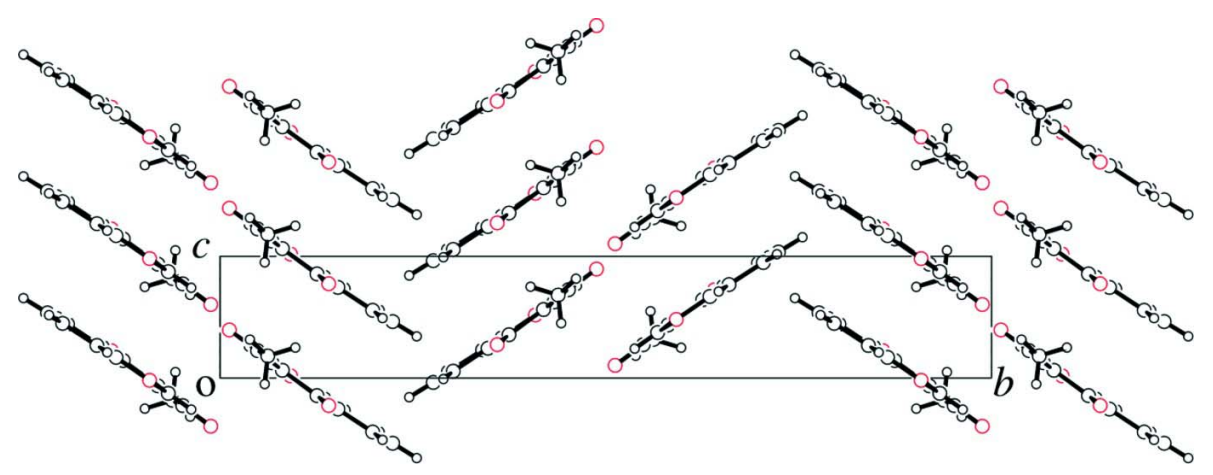

\section{Figure 3}

A view of the title compound down to the $a$-axis.

\section{3-Acetyl-4H-chromen-4-one}

Crystal data

$\mathrm{C}_{11} \mathrm{H}_{8} \mathrm{O}_{3}$

$M_{r}=188.18$

Monoclinic, $P 2_{1} / n$

Hall symbol: -P $2 \mathrm{yn}$

$a=8.016(13) \AA$

$b=25.93$ (6) $\AA$

$c=4.091(8) \AA$

$\beta=94.79(14)^{\circ}$

$V=847(3) \AA^{3}$

$Z=4$

\section{Data collection}

Rigaku AFC-7R

diffractometer

$\omega$ scans

2377 measured reflections

1962 independent reflections

1510 reflections with $F^{2}>2.0 \sigma\left(F^{2}\right)$

$R_{\text {int }}=0.018$

\section{Refinement}

Refinement on $F^{2}$

$R\left[F^{2}>2 \sigma\left(F^{2}\right)\right]=0.041$

$w R\left(F^{2}\right)=0.112$

$S=1.03$

1959 reflections

128 parameters

0 restraints

Primary atom site location: structure-invariant direct methods
$F(000)=392.00$

$D_{\mathrm{x}}=1.475 \mathrm{Mg} \mathrm{m}^{-3}$

Mo $K \alpha$ radiation, $\lambda=0.71069 \AA$

Cell parameters from 25 reflections

$\theta=15.2-17.5^{\circ}$

$\mu=0.11 \mathrm{~mm}^{-1}$

$T=100 \mathrm{~K}$

Prismatic, colorless

$0.42 \times 0.25 \times 0.20 \mathrm{~mm}$

$\theta_{\max }=27.6^{\circ}$

$h=-5 \rightarrow 10$

$k=0 \rightarrow 33$

$l=-5 \rightarrow 5$

3 standard reflections every 150 reflections intensity decay: $-0.5 \%$

Secondary atom site location: difference Fourier map

Hydrogen site location: inferred from neighbouring sites

$\mathrm{H}$-atom parameters constrained

$w=1 /\left[\sigma^{2}\left(F_{\mathrm{o}}{ }^{2}\right)+(0.0509 P)^{2}+0.3687 P\right]$ where $P=\left(F_{\mathrm{o}}^{2}+2 F_{\mathrm{c}}^{2}\right) / 3$

$(\Delta / \sigma)_{\max }<0.001$

$\Delta \rho_{\max }=0.31$ e $\AA^{-3}$

$\Delta \rho_{\min }=-0.20$ e $\AA^{-3}$

Special details

Refinement. Refinement was performed using all reflections. The weighted $R$-factor $(w R)$ and goodness of fit $(S)$ are based on $F^{2}$. $R$-factor (gt) are based on $F$. The threshold expression of $F^{2}>2.0 \sigma\left(F^{2}\right)$ is used only for calculating $R$-factor (gt). 
Fractional atomic coordinates and isotropic or equivalent isotropic displacement parameters $\left(\AA^{2}\right)$

\begin{tabular}{|c|c|c|c|c|}
\hline & $x$ & $y$ & $z$ & $U_{\text {iso }} * / U_{\text {eq }}$ \\
\hline $\mathrm{O} 1$ & $-0.18103(13)$ & $0.09136(4)$ & $1.0184(3)$ & $0.0256(3)$ \\
\hline $\mathrm{O} 2$ & $0.27801(13)$ & $0.14078(4)$ & 0.7749 & $0.0296(3)$ \\
\hline $\mathrm{O} 3$ & 0.22177 (14) & $0.01208(4)$ & $1.3956(4)$ & $0.0352(3)$ \\
\hline $\mathrm{C} 1$ & $-0.03986(18)$ & $0.06711(6)$ & $1.1256(4)$ & $0.0239(4)$ \\
\hline $\mathrm{C} 2$ & $0.11774(18)$ & $0.08077(5)$ & $1.0636(4)$ & $0.0212(3)$ \\
\hline $\mathrm{C} 3$ & $0.14176(18)$ & $0.12556(6)$ & $0.8560(4)$ & $0.0210(3)$ \\
\hline $\mathrm{C} 4$ & $-0.01216(18)$ & $0.19777(6)$ & $0.5570(4)$ & $0.0233(4)$ \\
\hline $\mathrm{C} 5$ & $-0.1584(2)$ & $0.22372(6)$ & $0.4646(4)$ & $0.0264(4)$ \\
\hline C6 & -0.31159 (19) & $0.20407(6)$ & $0.5507(4)$ & $0.0268(4)$ \\
\hline $\mathrm{C} 7$ & $-0.31830(18)$ & $0.15967(6)$ & 0.7318 & $0.0251(4)$ \\
\hline $\mathrm{C} 8$ & $-0.01438(17)$ & $0.15278(5)$ & $0.7452(4)$ & $0.0202(3)$ \\
\hline C9 & $-0.16842(18)$ & $0.13482(5)$ & $0.8296(4)$ & $0.0212(3)$ \\
\hline $\mathrm{C} 10$ & 0.25597 (19) & $0.04850(6)$ & $1.2256(4)$ & $0.0232(4)$ \\
\hline $\mathrm{C} 11$ & $0.43401(19)$ & $0.06180(6)$ & $1.1822(5)$ & $0.0270(4)$ \\
\hline H1 & -0.0510 & 0.0374 & 1.2581 & $0.0286^{*}$ \\
\hline $\mathrm{H} 2$ & 0.0912 & 0.2105 & 0.4924 & $0.0280^{*}$ \\
\hline $\mathrm{H} 3$ & -0.1554 & 0.2549 & 0.3429 & $0.0316^{*}$ \\
\hline $\mathrm{H} 4$ & -0.4124 & 0.2217 & 0.4826 & $0.0322 *$ \\
\hline H5 & -0.4223 & 0.1463 & 0.7889 & $0.0301 *$ \\
\hline H6A & 0.5082 & 0.0387 & 1.3165 & $0.0324 *$ \\
\hline H7B & 0.4557 & 0.0976 & 1.2506 & $0.0324 *$ \\
\hline $\mathrm{H} 8 \mathrm{C}$ & 0.4550 & 0.0579 & 0.9509 & $0.0324 *$ \\
\hline
\end{tabular}

Atomic displacement parameters $\left(\AA^{2}\right)$

\begin{tabular}{lllllll}
\hline & $U^{11}$ & $U^{22}$ & $U^{33}$ & $U^{12}$ & $U^{13}$ & $U^{23}$ \\
\hline O1 & $0.0172(5)$ & $0.0250(6)$ & $0.0351(6)$ & $-0.0015(4)$ & $0.0041(5)$ & $0.0048(5)$ \\
O2 & $0.0169(6)$ & $0.0302(6)$ & $0.0419(7)$ & $-0.0011(5)$ & $0.0043(5)$ & $0.0109(5)$ \\
O3 & $0.0269(6)$ & $0.0319(7)$ & $0.0468(8)$ & $0.0004(5)$ & $0.0032(6)$ & $0.0164(6)$ \\
C1 & $0.0211(7)$ & $0.0222(7)$ & $0.0282(8)$ & $-0.0004(6)$ & $0.0018(6)$ & $0.0013(6)$ \\
C2 & $0.0183(7)$ & $0.0202(7)$ & $0.0250(8)$ & $-0.0003(6)$ & $0.0018(6)$ & $-0.0014(6)$ \\
C3 & $0.0175(7)$ & $0.0213(7)$ & $0.0243(8)$ & $-0.0013(6)$ & $0.0026(6)$ & $-0.0023(6)$ \\
C4 & $0.0207(7)$ & $0.0231(7)$ & $0.0261(8)$ & $-0.0014(6)$ & $0.0015(6)$ & $-0.0012(6)$ \\
C5 & $0.0273(8)$ & $0.0234(8)$ & $0.0280(8)$ & $0.0021(6)$ & $-0.0001(7)$ & $0.0017(7)$ \\
C6 & $0.0205(8)$ & $0.0310(9)$ & $0.0285(8)$ & $0.0056(6)$ & $0.0000(6)$ & $-0.0010(7)$ \\
C7 & $0.0178(7)$ & $0.0293(8)$ & $0.0283(8)$ & $0.0001(6)$ & $0.0028(6)$ & $-0.0031(7)$ \\
C8 & $0.0175(7)$ & $0.0204(7)$ & $0.0227(8)$ & $-0.0003(6)$ & $0.0017(6)$ & $-0.0036(6)$ \\
C9 & $0.0192(7)$ & $0.0206(7)$ & $0.0239(8)$ & $-0.0007(6)$ & $0.0023(6)$ & $-0.0020(6)$ \\
C10 & $0.0217(8)$ & $0.0224(7)$ & $0.0253(8)$ & $0.0008(6)$ & $0.0017(6)$ & $0.0001(6)$ \\
C11 & $0.0193(8)$ & $0.0288(8)$ & $0.0327(9)$ & $0.0018(6)$ & $0.0011(6)$ & $0.0060(7)$ \\
& & & & & & \\
\hline
\end{tabular}

Geometric parameters $\left(A,{ }^{\circ}\right)$

\begin{tabular}{llll}
\hline $\mathrm{O} 1-\mathrm{C} 1$ & $1.336(3)$ & $\mathrm{C} 7-\mathrm{C} 9$ & $1.392(3)$ \\
$\mathrm{O} 1-\mathrm{C} 9$ & $1.375(3)$ & $\mathrm{C} 8-\mathrm{C} 9$ & $1.390(3)$
\end{tabular}




\begin{tabular}{|c|c|c|c|}
\hline $\mathrm{O} 2-\mathrm{C} 3$ & $1.233(3)$ & $\mathrm{C} 10-\mathrm{C} 11$ & $1.493(3)$ \\
\hline $\mathrm{O} 3-\mathrm{C} 10$ & $1.218(3)$ & $\mathrm{C} 1-\mathrm{H} 1$ & 0.950 \\
\hline $\mathrm{C} 1-\mathrm{C} 2$ & $1.356(3)$ & $\mathrm{C} 4-\mathrm{H} 2$ & 0.950 \\
\hline $\mathrm{C} 2-\mathrm{C} 3$ & $1.461(3)$ & $\mathrm{C} 5-\mathrm{H} 3$ & 0.950 \\
\hline $\mathrm{C} 2-\mathrm{C} 10$ & $1.498(3)$ & $\mathrm{C} 6-\mathrm{H} 4$ & 0.950 \\
\hline $\mathrm{C} 3-\mathrm{C} 8$ & $1.475(3)$ & $\mathrm{C} 7-\mathrm{H} 5$ & 0.950 \\
\hline $\mathrm{C} 4-\mathrm{C} 5$ & $1.377(3)$ & $\mathrm{C} 11-\mathrm{H} 6 \mathrm{~A}$ & 0.980 \\
\hline $\mathrm{C} 4-\mathrm{C} 8$ & $1.399(3)$ & $\mathrm{C} 11-\mathrm{H} 7 \mathrm{~B}$ & 0.980 \\
\hline $\mathrm{C} 5-\mathrm{C} 6$ & $1.401(3)$ & $\mathrm{C} 11-\mathrm{H} 8 \mathrm{C}$ & 0.980 \\
\hline $\mathrm{C} 6-\mathrm{C} 7$ & $1.373(4)$ & & \\
\hline $\mathrm{C} 1-\mathrm{O} 1-\mathrm{C} 9$ & $118.05(15)$ & $\mathrm{O} 3-\mathrm{C} 10-\mathrm{C} 11$ & $120.67(16)$ \\
\hline $\mathrm{O} 1-\mathrm{C} 1-\mathrm{C} 2$ & $126.32(18)$ & $\mathrm{C} 2-\mathrm{C} 10-\mathrm{C} 11$ & $119.82(17)$ \\
\hline $\mathrm{C} 1-\mathrm{C} 2-\mathrm{C} 3$ & $119.10(15)$ & $\mathrm{O} 1-\mathrm{C} 1-\mathrm{H} 1$ & 116.840 \\
\hline $\mathrm{C} 1-\mathrm{C} 2-\mathrm{C} 10$ & $115.94(17)$ & $\mathrm{C} 2-\mathrm{C} 1-\mathrm{H} 1$ & 116.843 \\
\hline $\mathrm{C} 3-\mathrm{C} 2-\mathrm{C} 10$ & $124.94(16)$ & $\mathrm{C} 5-\mathrm{C} 4-\mathrm{H} 2$ & 119.697 \\
\hline $\mathrm{O} 2-\mathrm{C} 3-\mathrm{C} 2$ & $125.01(15)$ & $\mathrm{C} 8-\mathrm{C} 4-\mathrm{H} 2$ & 119.697 \\
\hline $\mathrm{O} 2-\mathrm{C} 3-\mathrm{C} 8$ & $120.81(18)$ & $\mathrm{C} 4-\mathrm{C} 5-\mathrm{H} 3$ & 120.092 \\
\hline $\mathrm{C} 2-\mathrm{C} 3-\mathrm{C} 8$ & $114.17(16)$ & $\mathrm{C} 6-\mathrm{C} 5-\mathrm{H} 3$ & 120.097 \\
\hline $\mathrm{C} 5-\mathrm{C} 4-\mathrm{C} 8$ & $120.61(17)$ & $\mathrm{C} 5-\mathrm{C} 6-\mathrm{H} 4$ & 119.500 \\
\hline $\mathrm{C} 4-\mathrm{C} 5-\mathrm{C} 6$ & $119.81(19)$ & $\mathrm{C} 7-\mathrm{C} 6-\mathrm{H} 4$ & 119.487 \\
\hline $\mathrm{C} 5-\mathrm{C} 6-\mathrm{C} 7$ & $121.01(16)$ & $\mathrm{C} 6-\mathrm{C} 7-\mathrm{H} 5$ & 120.923 \\
\hline $\mathrm{C} 6-\mathrm{C} 7-\mathrm{C} 9$ & $118.14(17)$ & $\mathrm{C} 9-\mathrm{C} 7-\mathrm{H} 5$ & 120.937 \\
\hline $\mathrm{C} 3-\mathrm{C} 8-\mathrm{C} 4$ & $121.26(16)$ & $\mathrm{C} 10-\mathrm{C} 11-\mathrm{H} 6 \mathrm{~A}$ & 109.476 \\
\hline $\mathrm{C} 3-\mathrm{C} 8-\mathrm{C} 9$ & $120.79(17)$ & $\mathrm{C} 10-\mathrm{C} 11-\mathrm{H} 7 \mathrm{~B}$ & 109.464 \\
\hline $\mathrm{C} 4-\mathrm{C} 8-\mathrm{C} 9$ & $117.95(15)$ & $\mathrm{C} 10-\mathrm{C} 11-\mathrm{H} 8 \mathrm{C}$ & 109.473 \\
\hline $\mathrm{O} 1-\mathrm{C} 9-\mathrm{C} 7$ & $116.03(16)$ & $\mathrm{H} 6 \mathrm{~A}-\mathrm{C} 11-\mathrm{H} 7 \mathrm{~B}$ & 109.469 \\
\hline $\mathrm{O} 1-\mathrm{C} 9-\mathrm{C} 8$ & $121.52(15)$ & $\mathrm{H} 6 \mathrm{~A}-\mathrm{C} 11-\mathrm{H} 8 \mathrm{C}$ & 109.475 \\
\hline $\mathrm{C} 7-\mathrm{C} 9-\mathrm{C} 8$ & $122.45(17)$ & $\mathrm{H} 7 \mathrm{~B}-\mathrm{C} 11-\mathrm{H} 8 \mathrm{C}$ & 109.471 \\
\hline $\mathrm{O} 3-\mathrm{C} 10-\mathrm{C} 2$ & $119.50(17)$ & & \\
\hline $\mathrm{C} 1-\mathrm{O} 1-\mathrm{C} 9-\mathrm{C} 7$ & $-178.13(12)$ & $\mathrm{H} 2-\mathrm{C} 4-\mathrm{C} 5-\mathrm{H} 3$ & -2.1 \\
\hline $\mathrm{C} 1-\mathrm{O} 1-\mathrm{C} 9-\mathrm{C} 8$ & 1.35 (19) & $\mathrm{H} 2-\mathrm{C} 4-\mathrm{C} 8-\mathrm{C} 3$ & 2.2 \\
\hline $\mathrm{C} 9-\mathrm{O} 1-\mathrm{C} 1-\mathrm{C} 2$ & $-0.8(3)$ & $\mathrm{H} 2-\mathrm{C} 4-\mathrm{C} 8-\mathrm{C} 9$ & -178.8 \\
\hline $\mathrm{C} 9-\mathrm{O} 1-\mathrm{C} 1-\mathrm{H} 1$ & 179.2 & $\mathrm{C} 4-\mathrm{C} 5-\mathrm{C} 6-\mathrm{C} 7$ & $1.3(3)$ \\
\hline $\mathrm{O} 1-\mathrm{C} 1-\mathrm{C} 2-\mathrm{C} 3$ & $-1.2(3)$ & $\mathrm{C} 4-\mathrm{C} 5-\mathrm{C} 6-\mathrm{H} 4$ & -178.7 \\
\hline $\mathrm{O} 1-\mathrm{C} 1-\mathrm{C} 2-\mathrm{C} 10$ & $177.31(13)$ & $\mathrm{H} 3-\mathrm{C} 5-\mathrm{C} 6-\mathrm{C} 7$ & -178.7 \\
\hline $\mathrm{H} 1-\mathrm{C} 1-\mathrm{C} 2-\mathrm{C} 3$ & 178.9 & $\mathrm{H} 3-\mathrm{C} 5-\mathrm{C} 6-\mathrm{H} 4$ & 1.3 \\
\hline $\mathrm{H} 1-\mathrm{C} 1-\mathrm{C} 2-\mathrm{C} 10$ & -2.7 & $\mathrm{C} 5-\mathrm{C} 6-\mathrm{C} 7-\mathrm{C} 9$ & $0.4(3)$ \\
\hline $\mathrm{C} 1-\mathrm{C} 2-\mathrm{C} 3-\mathrm{O} 2$ & $-177.82(14)$ & $\mathrm{C} 5-\mathrm{C} 6-\mathrm{C} 7-\mathrm{H} 5$ & -179.6 \\
\hline $\mathrm{C} 1-\mathrm{C} 2-\mathrm{C} 3-\mathrm{C} 8$ & $2.4(2)$ & $\mathrm{H} 4-\mathrm{C} 6-\mathrm{C} 7-\mathrm{C} 9$ & -179.6 \\
\hline $\mathrm{C} 1-\mathrm{C} 2-\mathrm{C} 10-\mathrm{O} 3$ & $1.2(2)$ & $\mathrm{H} 4-\mathrm{C} 6-\mathrm{C} 7-\mathrm{H} 5$ & 0.4 \\
\hline $\mathrm{C} 1-\mathrm{C} 2-\mathrm{C} 10-\mathrm{C} 11$ & $-177.68(13)$ & $\mathrm{C} 6-\mathrm{C} 7-\mathrm{C} 9-\mathrm{O} 1$ & $178.20(13)$ \\
\hline $\mathrm{C} 3-\mathrm{C} 2-\mathrm{C} 10-\mathrm{O} 3$ & $179.57(13)$ & $\mathrm{C} 6-\mathrm{C} 7-\mathrm{C} 9-\mathrm{C} 8$ & $-1.3(3)$ \\
\hline $\mathrm{C} 3-\mathrm{C} 2-\mathrm{C} 10-\mathrm{C} 11$ & $0.7(3)$ & $\mathrm{H} 5-\mathrm{C} 7-\mathrm{C} 9-\mathrm{O} 1$ & -1.8 \\
\hline $\mathrm{C} 10-\mathrm{C} 2-\mathrm{C} 3-\mathrm{O} 2$ & $3.9(3)$ & $\mathrm{H} 5-\mathrm{C} 7-\mathrm{C} 9-\mathrm{C} 8$ & 178.7 \\
\hline $\mathrm{C} 10-\mathrm{C} 2-\mathrm{C} 3-\mathrm{C} 8$ & $-175.94(12)$ & $\mathrm{C} 3-\mathrm{C} 8-\mathrm{C} 9-\mathrm{O} 1$ & $0.0(2)$ \\
\hline $\mathrm{O} 2-\mathrm{C} 3-\mathrm{C} 8-\mathrm{C} 4$ & $-2.7(3)$ & $\mathrm{C} 3-\mathrm{C} 8-\mathrm{C} 9-\mathrm{C} 7$ & $179.50(12)$ \\
\hline
\end{tabular}


$\mathrm{O} 2-\mathrm{C} 3-\mathrm{C} 8-\mathrm{C} 9$

$\mathrm{C} 2-\mathrm{C} 3-\mathrm{C} 8-\mathrm{C} 4$

$\mathrm{C} 2-\mathrm{C} 3-\mathrm{C} 8-\mathrm{C} 9$

$\mathrm{C} 5-\mathrm{C} 4-\mathrm{C} 8-\mathrm{C} 3$

$\mathrm{C} 5-\mathrm{C} 4-\mathrm{C} 8-\mathrm{C} 9$

$\mathrm{C} 8-\mathrm{C} 4-\mathrm{C} 5-\mathrm{C} 6$

$\mathrm{C} 8-\mathrm{C} 4-\mathrm{C} 5-\mathrm{H} 3$

$\mathrm{H} 2-\mathrm{C} 4-\mathrm{C} 5-\mathrm{C} 6$
$178.31(13)$

$177.09(12)$

$-1.87(19)$

$-177.81(13)$

$1.2(2)$

$-2.0(3)$

177.9

178.0
$\mathrm{C} 4-\mathrm{C} 8-\mathrm{C} 9-\mathrm{O} 1$

$\mathrm{C} 4-\mathrm{C} 8-\mathrm{C} 9-\mathrm{C} 7$

$\mathrm{O} 3-\mathrm{C} 10-\mathrm{C} 11-\mathrm{H} 6 \mathrm{~A}$

$\mathrm{O} 3-\mathrm{C} 10-\mathrm{C} 11-\mathrm{H} 7 \mathrm{~B}$

$\mathrm{O} 3-\mathrm{C} 10-\mathrm{C} 11-\mathrm{H} 8 \mathrm{C}$

$\mathrm{C} 2-\mathrm{C} 10-\mathrm{C} 11-\mathrm{H} 6 \mathrm{~A}$

$\mathrm{C} 2-\mathrm{C} 10-\mathrm{C} 11-\mathrm{H} 7 \mathrm{~B}$

$\mathrm{C} 2-\mathrm{C} 10-\mathrm{C} 11-\mathrm{H} 8 \mathrm{C}$
$-178.94(12)$

$0.5(2)$

$-3.2$

$-123.2$

116.8

175.7

55.7

$-64.3$

Hydrogen-bond geometry $\left(\AA,{ }^{o}\right)$

\begin{tabular}{lllll}
\hline$D-\mathrm{H} \cdots A$ & $D-\mathrm{H}$ & $\mathrm{H} \cdots A$ & $D \cdots A$ & $D-\mathrm{H} \cdots A$ \\
\hline $\mathrm{C} 7-\mathrm{H} 5 \cdots \mathrm{O} 2^{\mathrm{i}}$ & 0.95 & 2.40 & $3.292(6)$ & 155 \\
$\mathrm{C} 1-\mathrm{H} 1 \cdots \mathrm{O} 3^{\mathrm{ii}}$ & 0.95 & 2.31 & $3.264(5)$ & 148 \\
\hline
\end{tabular}

Symmetry codes: (i) $x-1, y, z$; (ii) $-x,-y,-z+3$. 